\title{
New insights into the neurobiology, diagnosis, and treatment of mood disorders
}

\author{
Carlos A. Zarate Jr.* \\ Experimental Therapeutics and Pathophysiology Branch, Intramural Research Program of the National Institute of Mental Health, National Institutes of \\ Health, Section Neurobiology and Treatment of Mood Disorders, Bethesda, Maryland, USA
}

I am pleased to welcome readers to this special issue of CNS Spectrums on Mood Disorders. Geared toward clinicians, these articles highlight four important, recent concepts in neurobiology and clinical care in mood disorders; these range from genetics, to intracellular signaling cascades, to patients with anxious depression, and to the management of mood disorders in pregnancy. It is our hope that the comprehensive overview of the key concepts provided in this special issue will allow readers to draw important insights that inform their clinical practice.

In "Pharmacogenetics of mood disorders: what clinicians need to know," Dr. Laje reviews the extant knowledge regarding the evolving field of pharmacogenetics and discusses the use of pharmacogenetics in clinical care. The article introduces the basic principles of genetics and pharmacogenetics, and synthesizes research findings to date. Dr. Laje introduces readers to terms such as genome-wide association studies (GWAS), explains genome-wide and candidate gene studies, and then carefully explains how such studies might be used to decipher the underlying pathophysiology of mental disorders, or predict treatment response and adverse events. The article also describes the limitations associated with existing phenotypes, and how these might have stymied progress in identifying genes involved in illness or treatment outcome. Dr. Laje echoes our growing understanding that, regarding mood disorders, it is likely that our current diagnostic system culminates in a heterogeneous collection of disorders rather that a single disorder. Similarly, each underlying disorder probably does not result from a single gene, but rather a combination of separate genes, thus making it difficult to identify the underlying genes or neurobiological

\footnotetext{
Experimental Therapeutics and Pathophysiology Branch, Intramural Research Program of the National Institute of Mental Health, National Institutes of Health.

*Address for correspondence: Carlos A. Zarate, Jr., MD, Chief Experimental Therapeutics \& Pathophysiology Branch \& Section Neurobiology and Treatment of Mood Disorders, Division of Intramural Research Program, National Institute of Mental Health, 10 Center Drive, CRC, Unit 7 SE, Rm. 7-5342 Bethesda, MD 2089-1282, USA.

(Email: zaratec@mail.nih.gov)
}

substrates involved in making DSM diagnoses. Indeed, there is perhaps a "dilution effect," in that identifying bio-substrates gets lost in the sea of heterogeneity. Robin Murray, a psychiatrist from Kings College London, noted that a clinical psychiatric diagnosis "may be the mental end-stage of all sorts of different neurobiological disorders.... .It may be a little like a diagnosis of 'renal failure' which is the end-stage of all sorts of medical disorders from heart disease to toxic shock" (cited in Abbott $^{1}$ ). As a result, some have argued that looking for endophenotypes as proxies for mental disorders may ultimately be a better approach; these endophenotypes are closer to the genes, and thus their study would likely identify genes involved in the pathophysiology of a given illness.

Although we cannot yet routinely apply pharmacogenetics to daily practice, Dr. Laje's article clearly outlines the early indications that this technology will soon become an important part of the clinician's armamentarium. One obvious example is cytochrome and potential drug-drug interactions. Fortunately, most recent drugs entering the market have been designed to have a more favorable cytochrome profile. Nevertheless, a multitude of possible drug interactions may occur, and clinicians should vigilantly check existing databases when prescribing medications, particularly in patients with complex medication regimens. Genes that regulate the bioavailability of antidepressant drugs could also significantly impact response and tolerability. It is also important to note that genetic testing in order to avoid serious adverse events when initiating treatment is beginning to be more regularly implemented into clinical practice. Carbamazepine therapy-which is often prescribed to patients with bipolar disorder and those with other neuropsychiatric illnesses-provides an excellent example of how such genetic testing might work. Carbamazepine's more serious side effects include hypersensitivity reactions such as Stevens-Johnson syndrome (SJS) and toxic epidermal necrolysis (TEN); both can prove fatal. However, predisposition to these hypersensitivity reactions has been linked to the human leukocyte antigen (HLA) genotype, particularly in Asian patients. Because of the incredibly 
important practical implications of these findings, some hospitals are now requiring HLA genotyping for at-risk individuals before starting carbamazepine.

The paper by Ionescu and colleagues, "Defining anxious depression: a review of the literature," explores the important nosological problems surrounding the term "anxious depression." This is a particular concern because anxious depression has been associated with more recurrences, greater functional impairment, worse response to selective serotonin reuptake inhibitors (SSRIs), and increased suicide risk than non-anxious depression. The careful review of this body of work by Dr. Ionescu and colleagues identifies a multitude of definitions used in studies of individuals with anxious depression; understanding these varying definitions is critical to any replication of findings regarding worsened outcomes in individuals with anxious depression. Ionescu and colleagues summarize the various categorical and dimensional approaches that investigators have used to define anxious depression, and clearly highlight the manner in which the varied definitions subsequently led to a wide range of findings. Notably, the authors also demonstrate that, presently, the dimensional definition of anxious depression is the most easily implemented as well as the most clinically useful. They also underscore the key unknowns that remain as we move toward a more reliable definition of anxious depression. For instance, how stable is the definition of dimensional anxious depression from week to week? What are the prognostic implications of this subgrouping? Do neurobiological differences appear when using a dimensional versus a categorical approach to define anxious depression?

This highlights another important issue in the field of mood disorders-that of standardization. Standardization will become increasingly important in the coming years, and will enable researchers to integrate and share results with greater confidence. ${ }^{2}$ The National Institute of Mental Health (NIMH) recently highlighted the limitations of existing diagnostic categories, showing that they fail to align with the findings emerging from clinical neuroscience, genetics, and treatment response studies. Consequently, the research community is moving toward Research Diagnostic Domain Criteria (RDoc), a new classification framework for research on mental disorders, and the first step in a transformative effort to develop a neuroscience-based psychiatric classification system. ${ }^{3}$ The article by Dr. Ionescu and colleagues underscores the need for such a system, and discusses how identifying an anxious depressive construct could help identify the cognitive systems, neural circuits, and behavioral dimensions involved in this illness.

"The management of mood disorders in pregnancy: alternatives to antidepressants," by Richards and Payne, addresses the complexities associated with caring for pregnant women with mood disorders. Clinicians often do not know how to proceed in such challenging situations, and may scramble to find the latest information on treating mood disorders in pregnancy, or may even seek out specialized centers. They are often surprised to find very little published information on this topic, and no local sites that deal with this special population. Drs. Richards and Payne do an excellent job of summarizing key concepts regarding the management of mood disorders in pregnancy. Their nuanced discussion also describes the risks of untreated mood disorders for both mother and baby, the risks associated with unnecessary or excessive treatment, and the manner in which this at times functions as a doubleedged sword. Importantly, Drs. Richards and Payne provide a comprehensive summary of appropriate and available pharmacological treatments while simultaneously highlighting the dearth of knowledge in this area, as well as common misconceptions regarding the efficacy and safety of nonpharmacological treatments during pregnancy. Clearly, this is a topic where much more research is needed, and any findings in this area could have enormous public health implications.

Finally, the articles by Niciu and colleagues, "Second Messenger/Signal Transduction Pathways in Major Mood Disorders; Moving from Membrane to Mechanism of Action, Part I: Major Depressive Disorder and Part II: Bipolar Disorder" provide an important overview of second messenger/signal transduction pathway alterations in major depressive disorder and bipolar disorder, and emphasizes that the development of new and improved therapeutics for these disorders is directly targeting increasingly complex intracellular pathways. Regarding major depressive disorder, Dr. Niciu and colleagues highlight those pathways that have received considerable scientific interest in recent years, including cyclic AMP (cAMP), protein kinase A (PKA), cAMP response element binding protein (CREB), mitogen-activated protein kinase (MAPK), and other neurotrophinmediated pathways such as $\mathrm{p} 11$, glycogen synthase kinase 3 beta (GSK3 3 ), Wnt/Fz/Dvl, and NFkB/ $\Delta$ FosB. The article also discusses key targets for bipolar disorder, including protein kinase C (PKC) and GSK-3 $\beta$.

Notably, recognition that intracellular neuroplasticity is key to the mechanism of action of antidepressants and mood stabilizers arose partly because no immediate therapeutic effects were observed with acute alterations of local neurotransmitter levels. Building on this theme, Dr. Niciu and colleagues further describe targets for the development of novel therapeutics with a rapid onset of antidepressant action, including the mammalian target of rapamycin (mTOR), release of inhibition on local translation via elongation factor 
stimulation, and GSK-3 inhibition. This is a rapidly growing and enormously important development in the field of mood disorders. Indeed, many studies have now described rapid antidepressant effects occurring within a matter of hours in patients with treatmentresistant depression, in stark contrast to the 6 weeks or longer associated with traditional antidepressants. At the forefront of this work is the N-methyl-D-aspartate (NMDA) antagonist ketamine; as a result, an evergrowing body of work has been investigating the putative cellular and molecular targets that might be responsible for ketamine's rapid antidepressant effect. Notably, all of this work has taken place in a comparatively short period of time, in strong juxtaposition to the lengthy process of drug discovery/development that has operated until now.

We hope that our readers enjoy these contributions, and that they enrich their clinical practice.

\section{Disclosures}

Funding for this work was supported by the Intramural Research Program of the National Institute of Mental
Health, National Institutes of Health (IRP-NIMH-NIH), by a NARSAD Independent Investigator Award to CAZ, and by the Brain \& Behavior Mood Disorders Research Award to CAZ. Dr. Zarate is listed as a co-inventor on a patent application for the use of ketamine and its metabolites in major depression. Dr. Zarate has assigned his rights in the patent to the U.S. government but will share a percentage of any royalties that may be received by the government.

\section{References}

1. Abbott A. Psychiatric genetics: the brains of the family. Nature. 2008; 454(7201): 154-157.

2. Insel TR, Morris SE, Heinssen RK. Standardization, integration, and sharing-leveraging research investments. Biol Psychiatry. 2011; 70(1): 5-6.

3. Insel TR, Cuthbert B, Garvey M, et al. Research domain criteria (RDoC): toward a new classification framework for research on mental disorders. Am J Psychiatry. 2010; 167(7): 748-751. 